\title{
Multiobjective Optimization of Injection Molding Process Parameters for the Precision Manufacturing of Plastic Optical Lens
}

\author{
Junhui Liu, Xindu Chen, Zeqin Lin, and Shipu Diao \\ Mechanical and Electrical Engineering Institute, Guangdong University of Technology, Guangdong, China \\ Correspondence should be addressed to Xindu Chen; chenxindu@gdut.edu.cn
}

Received 14 June 2017; Revised 26 September 2017; Accepted 8 October 2017; Published 4 December 2017

Academic Editor: Emilio Jiménez Macías

Copyright (C) 2017 Junhui Liu et al. This is an open access article distributed under the Creative Commons Attribution License, which permits unrestricted use, distribution, and reproduction in any medium, provided the original work is properly cited.

\begin{abstract}
Injection molding process parameters (IMPP) have a significant effect on the optical performance and surface waviness of precision plastic optical lens. This paper presents a set of procedures for the optimization of IMPP, with haze ratio (HR) reflecting the optical performance and peak-to-valley $20\left(\mathrm{PV}_{20}\right)$ reflecting the surface waviness as the optimization objectives. First, the orthogonal experiment was carried out with the Taguchi method, and the results were analyzed by ANOVA to screen out the IMPP having a significant effect on the objectives. Then, the $3^{4}$ full-factor experiment was conducted on the key IMPP, and the experimental results were used as the training and testing samples. The BPNN algorithm and the M-SVR algorithm were applied to establish the mapping relationships between the IMPP and objectives. Finally, the multiple-objective optimization was performed by applying the nondominated sorting genetic algorithm (NSGA-II), with the built M-SVR models as the fitness function of the objectives, to obtain a Pareto-optimal set, which improved the quality of plastic optical lens comprehensively. Through the experimental verification on the optimization results, the mean prediction error (MPE) of $\mathrm{HR}$ and $\mathrm{PV}_{20}$ is $7.16 \%$ and $9.78 \%$, respectively, indicating that the optimization method has high accuracy.
\end{abstract}

\section{Introduction}

In recent years, with the rapid development of the photovoltaic industry and information technology, the demand for high-quality optical components has shown a sharp upward trend. Optical lens is an indispensable component of these systems, so it is particularly important to carry out an indepth study on this aspect. The conventional optical lens processing materials are generally made of glass. However, with the development of new polymer materials with features such as low density, impact resistance, and low cost, as well as the production technology, more and more optical lenses are being made of special resins. The manufacturing technology of plastic optical lens generally involves injection molding process and compression methods. The injection molding process can produce complex products, with the advantages of high efficiency, low cost, and automation, so it is mainly used for producing plastic optical lens. Although the production of plastic optical lens has these advantages, compared with the glass optical lens, it also has some shortcomings due to constraints from material characteristics and the injection molding process, such as low light transmittance, birefringence, moisture absorption, poor heat resistance, and low geometry dimensional accuracy. In order to further enhance the quality of plastic optical lenses, more suitable new materials need to be developed and a new injection molding process needs to be studied. For a specific type of plastic, the quality of its injection molding products is affected by the injection machine, mold, and IMPP; the IMPP may be adjusted easily and therefore it has been widely investigated by researchers.

In the field of plastic optical lens processing, many researchers mainly adopt the geometric accuracy and defects of the optical lens as the research objectives, putting forward the corresponding optimization method and successfully improving the size, shape, and surface accuracy of the optical lens. Pazos et al. [1] estimated the ultimate thickness of PC biconcave and biconvex lenses using the CAE tool and evaluated the quality of the optical lens based on the number and location of weld lines and air-traps. J. Wen and P. Wen 
[2], Bensingh et al. [3], and Shieh et al. [4] optimized PMMA aspheric lens, bi-aspheric lens, and large diameter aspheric plastic lens, respectively, through design of experiments (DOE) and numerical simulation, reducing the volumetric shrinkage of the lens effectively. With the linear shrinkage of a specific point of the optical lens as an optimization objective, $\mathrm{Hu}$ and Cui [5] studied the effect of holding pressure and gate size on the objective. Lu and Khim [6] and Spina et al. [7] investigated some effects of the molding conditions on the surface contours and birefringence patterns of the lens, using a statistical experimental analysis method and grey relational component analysis coupled to the Taguchi design. Taking light transmission, surface waviness, and surface finish as the optimization objectives, Tsai et al. [8] established linear, exponential, and nonlinear regression models for the objectives and predicted the optimal IMPP; they concluded that the nonlinear regression model had higher prediction accuracy. They established an inverse model of injection molding for form accuracy of optical lens by combining the artificial neural network (ANN) with a genetic algorithm (GA) [9]. They also investigated the correlation between melt pressure and cavity pressure in different runner positions [10]. Other researchers, taking the optical and mechanical performance of the optical lens as the optimization objectives, proposed different methods to adjust the IMPP, reducing the birefringence and stress of the optical lens effectively. They mainly focused on the optimization of birefringence, used the numerical simulation method to study a PMMA CD-ROM lens [11], and verified the simulation results of a cyclic-olefincopolymers aspheric lens by combining with the Taguchi method [12]. Through the adjustment of IMPP, the birefringence of the optical lens was effectively reduced. Using the shortening of the processing cycle time and reduction of optical retardation as the optimization objectives, Turng et al. [13] achieved the high-quality PMMA lens via CAE simulation and experimental verification and evaluated the generated stresses and birefringence.

As mentioned above, although some researchers studied multiple quality objectives in the existing optimization of the plastic optical lens processing, they separately studied each individual objective while determining the optical IMPP, without considering the mutual effect of multiple objectives. However, the overall quality of the optical lens is obviously determined by its geometric accuracy, optical performance, and mechanical performance. Only optimizing the IMPP of an individual objective may lead to large defects in other optical quality objectives. From the practical point of view, therefore, it is necessary to optimize multiple quality objectives of the optical lens at the same time. The multiobjective optimization problem (MOP) has been widely used in the optimization of injection molding process and machine. For injection molding machine, Kuo and Chang [14] proposed a turbo injection mode (TIM) for an axial flux motor, and the particle swarm optimization (PSO) was used to find out the multiobjective optimal design solution. The product quality, energy-saving efficiency, and molding efficiency were optimized, and the corresponding multiple-objective Pareto-optimal set was obtained. Zhao et al. [15] took warp, volumetric shrinkage, and sink marks as the optimization objectives and achieved the multiobjective optimization by establishing the Kriging surrogate model and mapping the nonlinear relationships between the IMPP and objectives, using the NSGA-II algorithm. Yin et al. [16] presented an investigation on birefringence and warpage of thermoplastic lenses using microinjection molding, and the simultaneous control of both properties was accomplished through multiobjective optimization using the desirability function approach. Wang et al. [17] proposed a two-stage optimization approach to improve heating efficiency and temperature distribution uniformity for a new water-assisted rapid heat cycle molding, using RSM and the NSGA-II algorithm. The multiobjective optimization method mentioned above can be called model-based optimization (MBO) methods, and the quality of models plays an important role in the optimization results. In addition, there is another important multiobjective optimization method called model-free optimization (MFO), which integrates the simplex method. The MFO has been successfully applied to control and optimize part weight, part dimensions, and the focal length of molded products. Kong et al. [18] and Zhu et al. [19] proposed a novel MFO method for batch processes with short cycle times and low operational costs. In order to eliminate defects such as shrinkage and flash, Yang et al. [20] proposed a systematic method that combined digital image processing and MFO to solve such problems, and the MFO used online measurement as feedback to determine the optimal settings.

In order to obtain high-quality plastic optical lens, this paper takes the surface waviness and optical performance into account and presents a set of technical procedures for optimization of IMPP, providing reference for manufacturing high-quality optical lens. The existing literature contained the studies on the optimization of IMPP in terms of birefringence and light transmittance of plastic optical lens. In this paper, another important optical quality indicator, haze ratio (HR), which has not been studied, is one of the optimization objectives. It refers to the percentage of the intensity of transmitted light deviating from the incident light by $2.5^{\circ}$ in the overall transmitted light intensity. The greater the HR is, the more significantly lens' gloss and transparency will decrease. Meanwhile, another indicator reflecting the surface waviness, namely, peak-to-valley $20\left(\mathrm{PV}_{20}\right)$, was also taken as the optimization objective. The technical procedures adopted in this study are shown in Figure 1. First, the DOE was conducted, and then the key IMPP having significant effect on the two quality objectives were screened out via the Taguchi method combined with ANOVA, and the optimal combination of IMPP was initially determined. After that, a full factorial experiment was performed by the key IMPP. In order to obtain the nonlinear mapping relationships between the key IMPP and objectives, this paper used the data of the full factorial experiment as the sample, adopted multioutput support vector machine for regression (M-SVR) algorithm and the back-propagation neural network (BPNN) algorithm, and compared their prediction accuracies. Finally, the NSGA-II algorithm was used to optimize the two objectives, and a Pareto-optimal set was obtained and verified through experiment. 


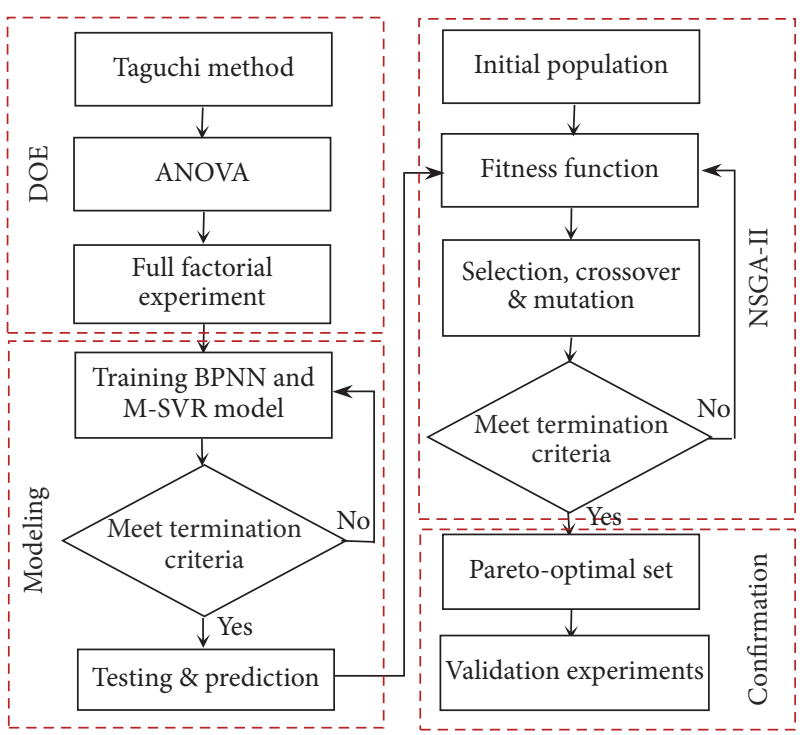

FIgURE 1: Flowchart of multiobjective optimization.

\section{Optical Lens and Experimental Setup}

Plastic optical lens needs to meet higher manufacturing requirements compared to that of common injection molding products, especially the surface waviness, and it must reach certain optical performance. Therefore, the equipment and process used for the manufacture of optical lens shall also meet more stringent requirements. Any large deviation in the product will seriously affect the quality of the lens. The lens studied in this paper, as shown in Figure 2, has a diameter of $20 \mathrm{~mm}$, and its material is PMMA-HT50Y made by SUMITOMO.

The precise injection machine adopts ARBURG ALLROUNDER 307 S 600-100. Its maximum injection weight is 230 grams, and the maximum injection pressure is $230 \mathrm{MPa}$. In this paper, the experiment on the lens involves $\mathrm{HR}$ and $\mathrm{PV}_{20}$, in which the haze ratio measurement device is the WGT-S transparent/haze tester, with the measurement accuracy of up to $0.01 \%$; and the $\mathrm{PV}_{20}$ measurement device is the Bruker-Contour GT-X3 three-dimensional surface profiler, which has the measurement accuracy of up to $0.1 \mathrm{~nm}$ and can accurately measure $\mathrm{PV}_{20}$, which is the average of the ten top points minus the ten lowest points on the wave surface in the measurement area. Evaluating the surface waviness with $\mathrm{PV}_{20}$ can eliminate the errors caused by some anomalous points or defects in the test data to a certain extent.

\section{Optimization Methodologies}

The optimization of precision injection molding process needs to be carried out generally by DOE. Due to the high-standard requirement of product quality, it is difficult to meet the requirement through only conducting trialand-error experiment by experienced technicians for long time. This has the characteristics of lack of planning and time consuming. The optimization of optical lens mainly relies on DOE, which has overcome the shortcomings of
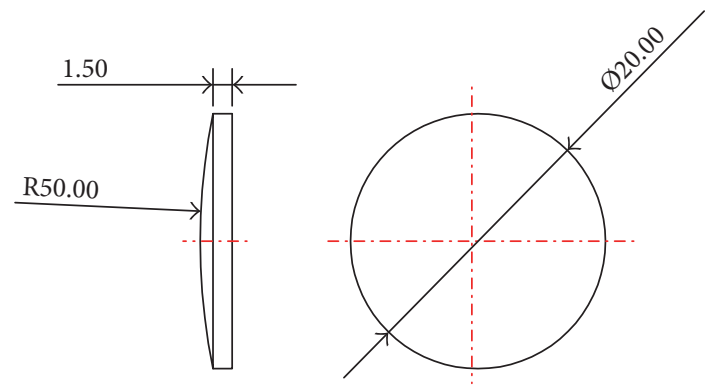

FIGURE 2: Dimensions of optical lens.

the trial-and-error method. If there are multiobjective optimization requirements to the product, especially when the IMPP are conflicting with each other, it is hard to obtain the global optimal solution only by DOE. Therefore, the intelligent algorithm was introduced on the basis of DOE. A set of optimization procedures for manufacturing plastic optical lens has been presented, as shown in Figure 1. The study conducted the double-objective optimization on the $\mathrm{HR}$ and $\mathrm{PV}_{20}$ to obtain the optimum IMPP for precise injection molding. The optimization process was divided into four stages. In the first stage, the ANOVA was carried out by using the statistical tool Minitab 15 with the Taguchi method as the DOE, in order to screen out the IMPP having a great influence on the two objectives, and then significant IMPP were obtained for full factorial experiments. In the second stage, the M-SVR and BPNN models were built and then trained and tested according to the data from full factorial experiments, to obtain the mapping relationships between key IMPP and objectives. In the third stage, a Pareto-optimal set was obtained through selection, crossover, and mutation, with the mapping relationships constructed by M-SVR as the fitness function of the NSGA-II algorithm. In the last stage, the obtained optimum IMPP were tested and verified to determine the effectiveness of the optimization method.

3.1. Taguchi Method. The $\mathrm{HR}$ and $\mathrm{PV}_{20}$ of plastic optical lens are affected by many IMPP. In order to study these indicators effectively and easily, the IMPP having little influence on the objectives need to be excluded using DOE. Taguchi method is an excellent DOE method. By arranging fewer experiments, we can obtain the influence degrees of all IMPP on the optimization objective, with combination of ANOVA. Taguchi method also introduces the concept of Signal to Noise ratio ( $\mathrm{S} / \mathrm{N}$ ratio), depending on which, we can ensure the product has stable quality and small fluctuation and is not sensitive to noise during production. The smaller the two optimization objectives are, the higher the quality of the lens will be, showing a smaller-the-better (STB) characteristic. The $\mathrm{S} / \mathrm{N}$ ratio of the STB is defined as [21]

$$
\eta_{\text {STB }}=-10 \times \lg \left(\frac{1}{n} \sum_{i=1}^{n} y_{i}^{2}\right)(\mathrm{dB}) \text {, }
$$

where $y_{i}$ is the response and $n$ is the number of replications. According to the calculated average value of the $\mathrm{S} / \mathrm{N}$ ratio for IMPP at each level, we can determine their optimum 


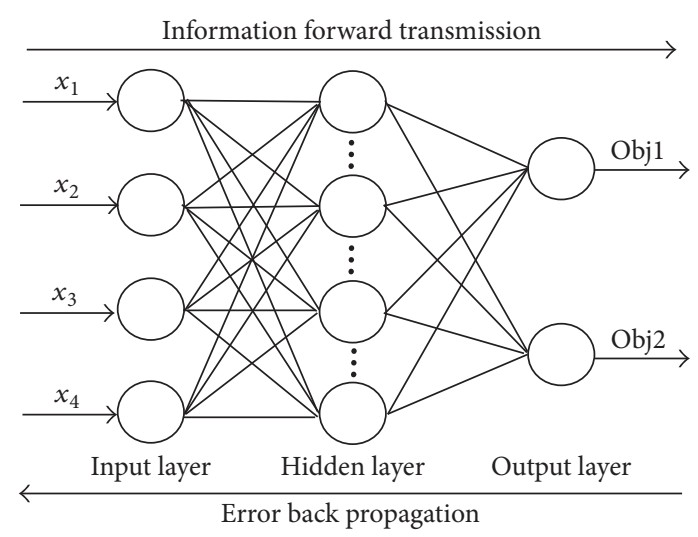

FIGURE 3: Architecture of the ANN.

level and finally obtain the optimum level combination. It should be noted that only a few discrete levels were selected for the experiment, so the optimization scheme obtained by the Taguchi method is not necessarily the global optimum. However, the initial optimized results can be used to adjust the range of process parameters, which can provide reference for further intelligent algorithm optimization. In order to verify the validity of the Taguchi method, the optimal combination was obtained according to $\mathrm{S} / \mathrm{N}$ ratio and then used to conduct the experiment. Compare the results after optimization with the previous ones. If the two objectives are smaller, it indicates that the Taguchi method is effective. Otherwise, it is necessary to reselect the IMPP to repeat the experiment.

3.2. BPNN Algorithm and M-SVR Algorithm. The ANN is an information processing method developed from inspiration of the biological nervous system. The network model built through the study of samples and analysis of the data relations has the prediction ability. The BPNN algorithm was first proposed by Rumelhart et al. [22], and it is a representative neural network, involving back propagation of errors and forward propagation of information. With the BPNN algorithm, the nonlinear relationships between the objectives and IMPP can be established. In this paper, a three-layer network structure was built, as shown in Figure 3; the output of each neuron is equal to the sum of the weights of all neurons from the previous layer plus a bias, and then the activation function generated the output

$$
O_{j}=f\left(\sum_{i=1}^{n}\left(w_{i j} \cdot x_{i}\right)+\theta_{j}\right)
$$

where $O_{j}$ is the output of the $j$ th neuron of the hidden layer or the output layer, $w_{i j}$ is the network weight from the $i$ th neuron to the $j$ th neuron, $x_{i}$ is the output of the $i$ th neuron, and $\theta_{j}$ is the bias of the $j$ th neuron. $f(\cdot)$ is an activation function of the hidden layer and the output layer, and a sigmoid function is selected as follows:

$$
f\left(O_{j}\right)=\frac{1-e^{-\left(\lambda O_{j}\right)}}{1+e^{-\left(\lambda O_{j}\right)}}
$$

where $\lambda$ is the gain factor of neurons and $f(\cdot)$ is within the range of $(-1,1)$. The model gradually adjusts the network weight by minimizing the actual output and network output

$$
\begin{aligned}
\Delta w_{i j}(n) & =\eta e(n) x_{j}(n), \\
w_{i j}(n+1) & =w_{i j}(n)+\Delta w_{i j}(n),
\end{aligned}
$$

where $e(n)$ is the difference between the actual output and the network output and $\eta$ is the learning rate. As the BPNN algorithm is based on the gradient descent method, there are some shortcomings such as large computation burden, long training time, demand for a large number of samples, and slow convergence. In this paper, the small number of experiments and training samples may result in that the prediction ability of BPNN is not so strong. Therefore, the M-SVR algorithm was also adopted to establish a nonlinear model in terms of objectives and IMPP, so that we could compare the prediction ability of the two algorithms.

Pérez-Cruz et al. [23] improved the SVR algorithm for single objective and obtained the M-SVR algorithm which can handle multiple output objectives. They used the iterative reweight least square (IRWLS) method to solve the dual problem by redefining the $\varepsilon$-insensitive loss function in the hyperspherical space and finally obtained the prediction model of each output terminal. It is a type of effective method for Multi-Input Multi-Output (MIMO) modelling of small samples.

Suppose that the given sample data is $D_{x, y}=\left\{\left(x_{i}, y_{i}\right)\right.$ | $i=1,2, \ldots, L\}, x_{i} \in \mathbf{R}^{N}$, map the input data $x_{i}$ into the highdimensional Hilbert space, and the mapped data $\varphi\left(x_{i}\right) \in \mathbf{R}^{f}$ may be obtained. The problem to be solved by SVR is how to select vectors $\mathbf{W}$ and $\mathbf{b}$, so that the regression results obtained from $\widetilde{y_{i}}=\emptyset\left(x_{i}\right) \mathbf{W}+\mathbf{b}$ have the minimum error, where $\mathbf{W}=$ $\left[\mathbf{w}^{1} \mathbf{w}^{2} \cdots \mathbf{w}^{N}\right]$ is a $f \times N$ dimensional matrix, $\mathbf{w}^{j}$ is a column vector, $\mathbf{b}=\left[\mathbf{b}^{1} \mathbf{b}^{2} \cdots \mathbf{b}^{N}\right]$ is a row vector, $\mathbf{b}^{j}(j=1, \ldots, N)$ is a constant, and $\emptyset\left(x_{i}\right)$ is a nonlinear mapping function. Thus, the minimized objective function $L_{p}$ is established to solve $\mathbf{W}$ and $\mathbf{b}$ :

$$
\min _{\mathbf{w}, \mathbf{b}} L_{p}=\frac{1}{2} \sum_{j=1}^{N}\left\|\mathbf{w}^{j}\right\|^{2}+C \sum_{i=1}^{L} L\left(u_{i}\right)
$$

The $\varepsilon$-insensitive loss function can be extended into a multidimensional form. Supersede the original singledimensional error with $L_{2}$ norm of the output variable error, and $L\left(u_{i}\right)$ was defined as

$$
L\left(u_{i}\right)= \begin{cases}0, & u<\varepsilon, \\ u^{2}-2 u \varepsilon+\varepsilon^{2}, & u \geq \varepsilon,\end{cases}
$$

where $u_{i}=\left\|e_{i}\right\|=\sqrt{e_{i}^{T} e_{i}}, e_{i}=y_{i}-\widetilde{y}_{i}, \varepsilon$ is the allowable deviation, and $C$ is the penalty factor. Through the first-order 
TABLE 1: The IMPP and their levels for the Taguchi experiment.

\begin{tabular}{|c|c|c|c|}
\hline IMPP & Level 1 & Level 2 & Level 3 \\
\hline$A$ : melt temperature $\left({ }^{\circ} \mathrm{C}\right)$ & 220 & 230 & \\
\hline$B$ : injection velocity $(\mathrm{mm} / \mathrm{s})$ & 60 & 70 & 80 \\
\hline$C$ : injection pressure $(\mathrm{MPa})$ & 80 & 90 & 100 \\
\hline$D$ : VP switchover (mm) & 5.2 & 5.4 & 5.6 \\
\hline$E$ : packing time (s) & 4 & 6 & 8 \\
\hline$F$ : packing pressure $(\mathrm{MPa})$ & 90 & 100 & 110 \\
\hline$G$ : mold temperature $\left({ }^{\circ} \mathrm{C}\right)$ & 60 & 75 & 90 \\
\hline$H$ : cooling time $(\mathrm{s})$ & 15 & 25 & 35 \\
\hline
\end{tabular}

Taylor expansion, the approximate quadratic programming form of Formula (5) is obtained:

$$
\begin{aligned}
L_{p}^{\prime}(\mathbf{W}, \mathbf{b}) & =\frac{1}{2} \sum_{j=1}^{N}\left\|\mathbf{w}^{j}\right\|^{2}+\frac{1}{2} \sum_{i=1}^{L} a_{i} u_{i}^{2}+C T, \\
a_{i} & =\left\{\begin{array}{lc}
0, & u_{i}^{k}<\varepsilon, \\
\frac{2 C\left(u_{i}^{k}-\varepsilon\right)}{u_{i}^{k}}, & u_{i}^{k} \geq \varepsilon,
\end{array}\right. \\
T & =\sum_{i=1}^{L} L\left(u_{i}^{k}\right)-\left.\frac{d L(u)}{d u}\right|_{u_{i}^{k}} \frac{\left(u_{i}^{k}\right)^{2}}{2 u_{i}^{k}},
\end{aligned}
$$

where $a_{i}$ and $T$ are the constants independent of $\mathbf{W}, \mathbf{b}$, and then, we can minimize $L_{p}^{\prime}(\mathbf{W}, \mathbf{b})$ with the IRWLS method to calculate the optimal solution $\mathbf{W}_{\mathbf{s}}, \mathbf{b}_{\mathbf{s}}$. Finally, the relation between input and output is obtained:

$$
\widetilde{y_{i}}=\emptyset\left(x_{i}\right) \mathbf{W}_{\mathbf{s}}+\mathbf{b}_{\mathbf{s}} .
$$

In order to evaluate the ability of BPNN and M-SVR models of fitting and predicting, the coefficient of determination $\left(R^{2}\right)$ was used for analysis in this paper. The closer $R^{2}$ is to 1 , the more data the model can interpret, and the better the model performance will be. $R^{2}$ is defined as follows:

$$
R^{2}=\frac{\mathrm{ESS}}{\mathrm{TSS}}=1-\frac{\mathrm{RSS}}{\mathrm{TSS}}
$$

where ESS is the error sum of squares, TSS is the total sum of squares, and RSS is the regression sum of squares, and they satisfy the relation TSS $=$ ESS + RSS.

3.3. NSGA-II Algorithm. Since MOP often involves optimizing several objectives with trade-off relationships, it has no solution that can achieve the optimization of all objectives at the same time. The purpose of the multiobjective optimization algorithm is to obtain a representative Pareto-optimal set in the decision space, so that their distribution in the objective space Pareto front is broad and uniform as much as possible. There are different algorithms to solve MOP; the most widely used one is the NSGA-II algorithm developed by Deb et al. [24]. The algorithm enables individuals in a Paretooptimal set to extend into the entire objective space uniformly through the introduction of the fast nondominated sorting algorithm and the elite retention strategy, which ensures the diversity of the population. The basic steps of the NSGA-II algorithm are as follows.

Step 1. Initialize parameters, such as population size, control variable limits, crossover and mutation probability, and termination criteria.

Step 2. Nondominated sorting: sort the population according to nondomination criteria.

Step 3. Calculate the crowding distance for each individual.

Step 4. Execute the genetic operator. Generate the intermediate population by selection, crossover, and mutation operators.

Step 5. Selection and combination: consolidate descendant and the contemporary populations; the descendant individuals are reset through rank and crowding distance.

Step 6. Return to Step 2 and repeat until termination criteria are reached.

\section{Result and Discussion}

4.1. Screening Significant IMPP. There may be a number of IMPP that can affect the quality of the injection products. Taking all of them into consideration to carry out experiments means a very large workload, and it is not necessary. We can conduct the screening experiment on some IMPP that may have significant effect on the product quality, according to our production practice experience. In this paper, a total of eight IMPP were selected for experiments, including melt temperature, injection velocity, injection pressure, VP switchover, packing time, packing pressure, molding temperature, and cooling time. The Taguchi method was taken as the DOE method for the $L_{18}\left(2^{1} \times 3^{7}\right)$ orthogonal experiment. The diameter of the optical lens is $20 \mathrm{~mm}$, which belongs to a small size product. In order to improve production efficiency, six cavities of the same size have been designed. According to the suggestion of PMMA-HT50Y supplier, the number of cavities, and the size of the product, the levels of IMPP should be initially set out as shown in Table 1 .

In order to reduce the experimental and measurement errors, each experimental treatment was carried out twice, 
TABLE 2: Experimental results and $\mathrm{S} / \mathrm{N}$ ratio of $\mathrm{HR}$ and $\mathrm{PV}_{20}$.

\begin{tabular}{|c|c|c|c|c|c|c|c|c|c|c|c|c|}
\hline \multirow{2}{*}{ Number } & \multicolumn{8}{|c|}{ IMPP } & \multirow{2}{*}{ HR (\%) } & \multirow{2}{*}{$\mathrm{PV}_{20}(\mu \mathrm{m})$} & \multicolumn{2}{|c|}{$\mathrm{S} / \mathrm{N}$ ratio $(\mathrm{dB})$} \\
\hline & $A$ & $B$ & C & $D$ & $E$ & $F$ & $G$ & $H$ & & & HR & $\mathrm{PV}_{20}$ \\
\hline (1) & 230 & 60 & 90 & 5.2 & 4 & 110 & 90 & 25 & 8.23 & 1.102 & -18.3080 & -0.8436 \\
\hline (2) & 220 & 70 & 90 & 5.4 & 8 & 110 & 60 & 15 & 5.31 & 1.721 & -14.5019 & -4.7156 \\
\hline (3) & 220 & 80 & 100 & 5.2 & 8 & 100 & 60 & 25 & 6.06 & 0.813 & -15.6495 & 1.7982 \\
\hline (4) & 220 & 60 & 100 & 5.6 & 8 & 110 & 90 & 35 & 8.85 & 0.871 & -18.9389 & 1.1996 \\
\hline (5) & 230 & 80 & 100 & 5.4 & 4 & 100 & 90 & 15 & 12.22 & 1.41 & -21.7414 & -2.9844 \\
\hline (6) & 220 & 80 & 90 & 5.6 & 6 & 90 & 90 & 15 & 11.87 & 1.523 & -21.4890 & -3.6540 \\
\hline (7) & 220 & 60 & 90 & 5.4 & 6 & 100 & 75 & 25 & 6.05 & 0.321 & -15.6351 & 9.8699 \\
\hline (8) & 220 & 70 & 100 & 5.6 & 4 & 90 & 75 & 25 & 8.67 & 0.512 & -18.7604 & 5.8146 \\
\hline (9) & 220 & 60 & 80 & 5.2 & 4 & 90 & 60 & 15 & 4.85 & 1.825 & -13.7148 & -5.2253 \\
\hline (10) & 230 & 80 & 80 & 5.6 & 6 & 110 & 60 & 25 & 4.65 & 0.995 & -13.3491 & 0.0435 \\
\hline (11) & 230 & 60 & 80 & 5.6 & 8 & 100 & 75 & 15 & 6.32 & 0.804 & -16.0143 & 1.8949 \\
\hline (12) & 230 & 60 & 100 & 5.4 & 6 & 90 & 60 & 35 & 7.89 & 1.381 & -17.9415 & -2.8039 \\
\hline (13) & 220 & 70 & 80 & 5.2 & 6 & 100 & 90 & 35 & 8.31 & 0.482 & -18.3920 & 6.3391 \\
\hline (14) & 220 & 80 & 80 & 5.4 & 4 & 110 & 75 & 35 & 4.01 & 0.782 & -12.0629 & 2.1359 \\
\hline (15) & 230 & 70 & 90 & 5.6 & 4 & 100 & 60 & 35 & 6.11 & 0.873 & -15.7208 & 1.1797 \\
\hline (16) & 230 & 80 & 90 & 5.2 & 8 & 90 & 75 & 35 & 8.34 & 0.672 & -18.4233 & 3.4526 \\
\hline (17) & 230 & 70 & 80 & 5.4 & 8 & 90 & 90 & 25 & 9.68 & 1.363 & -19.7175 & -2.6899 \\
\hline (18) & 230 & 70 & 100 & 5.2 & 6 & 110 & 75 & 15 & 7.02 & 0.945 & -16.9267 & 0.4914 \\
\hline
\end{tabular}

TABLE 3: ANOVA for HR.

\begin{tabular}{|c|c|c|c|c|c|}
\hline Source & DF & Seq SS & Adj MS & $F$ & $P$ \\
\hline$A$ & 1 & 2.3328 & 2.3328 & 6.66 & 0.123 \\
\hline$B$ & 2 & 2.0707 & 1.0353 & 2.96 & 0.253 \\
\hline C & 2 & 14.1467 & 7.0733 & 20.20 & 0.047 \\
\hline$D$ & 2 & 1.1463 & 0.5732 & 1.64 & 0.379 \\
\hline E & 2 & 0.2569 & 0.1284 & 0.37 & 0.732 \\
\hline$F$ & 2 & 14.6025 & 7.3013 & 20.85 & 0.046 \\
\hline$G$ & 2 & 54.0143 & 27.0072 & 77.12 & 0.013 \\
\hline$H$ & 2 & 1.9299 & 0.9649 & 2.76 & 0.266 \\
\hline Error & 2 & 0.7004 & 0.3502 & & \\
\hline Total & 17 & 91.2006 & & & \\
\hline
\end{tabular}

totaling 36 experiments. At the same time, the experiments were arranged in random order to eliminate the interference of some hidden factors. The mold has six identical cavities. To ensure the tested data can reflect the whole batch of optical lens, this paper adopted a sequential sampling method. After each injection, one of optical lenses was selected to a box marked with serial numbers. Then, the next optical lens was taken in clockwise direction. The mean of the experimental results and the $\mathrm{S} / \mathrm{N}$ ratio of the two objectives, $\mathrm{HR}$ and $\mathrm{PV}_{20}$, are shown in Table 2. ANOVA of $\mathrm{HR}$ and $\mathrm{PV}_{20}$ is shown in Tables 3 and 4, respectively. The results show that the corresponding $P$ value of some IMPP is not more than 0.05, indicating it has a significant effect on the objectives, which must be considered during the optimization. The IMPP which have a significant effect on HR include injection pressure, packing pressure, and molding temperature. The IMPP which have a significant effect on $\mathrm{PV}_{20}$ include packing pressure, molding temperature, and cooling time.
$\mathrm{HR}$ and $\mathrm{PV}_{20}$ have the smaller-the-better characteristic; the main effects plots for $\mathrm{S} / \mathrm{N}$ ratios are shown in Figures 4 and 5, respectively, which further show the degrees effect of each process parameter on the objectives. At the same time, the initial optimization results of the Taguchi method were obtained, and the minimum process parameter combinations of $\mathrm{HR}$ and $\mathrm{PV}_{20}$ are $A=220, B=60, C=80, D=5.2, E=4$, $F=110, G=60, H=35$ and $A=220, B=70, C=90, D=$ $5.6, E=6, F=100, G=75, H=25$, respectively. In order to verify the initial optimization effect of the Taguchi method on the objectives, the experiments were conducted based on the initial optimization results, and the mean values of $\mathrm{HR}$ and $\mathrm{PV}_{20}$ were $4.36 \%$ and $0.303 \mu \mathrm{m}$, respectively. In comparison with the results in Table 2, the optimized objective is minimal, indicating that the Taguchi method has played a role in the initial optimization.

4.2. Building BPNN and M-SVR Model. The optimization on objectives using the Taguchi method cannot guarantee 
TABLE 4: ANOVA for $\mathrm{PV}_{20}$.

\begin{tabular}{|c|c|c|c|c|c|}
\hline Source & DF & Seq SS & Adj MS & $F$ & $P$ \\
\hline$A$ & 1 & 0.02683 & 0.02683 & 2.46 & 0.257 \\
\hline$B$ & 2 & 0.01487 & 0.00744 & 0.68 & 0.594 \\
\hline C & 2 & 0.01009 & 0.00505 & 0.46 & 0.683 \\
\hline$D$ & 2 & 0.18475 & 0.09237 & 8.48 & 0.105 \\
\hline E & 2 & 0.06436 & 0.03218 & 2.95 & 0.253 \\
\hline$F$ & 2 & 0.57191 & 0.28595 & 26.25 & 0.037 \\
\hline G & 2 & 1.15916 & 0.57958 & 53.20 & 0.018 \\
\hline$H$ & 2 & 1.09882 & 0.54941 & 50.43 & 0.019 \\
\hline Error & 2 & 0.02179 & 0.01089 & & \\
\hline Total & 17 & 3.15258 & & & \\
\hline
\end{tabular}

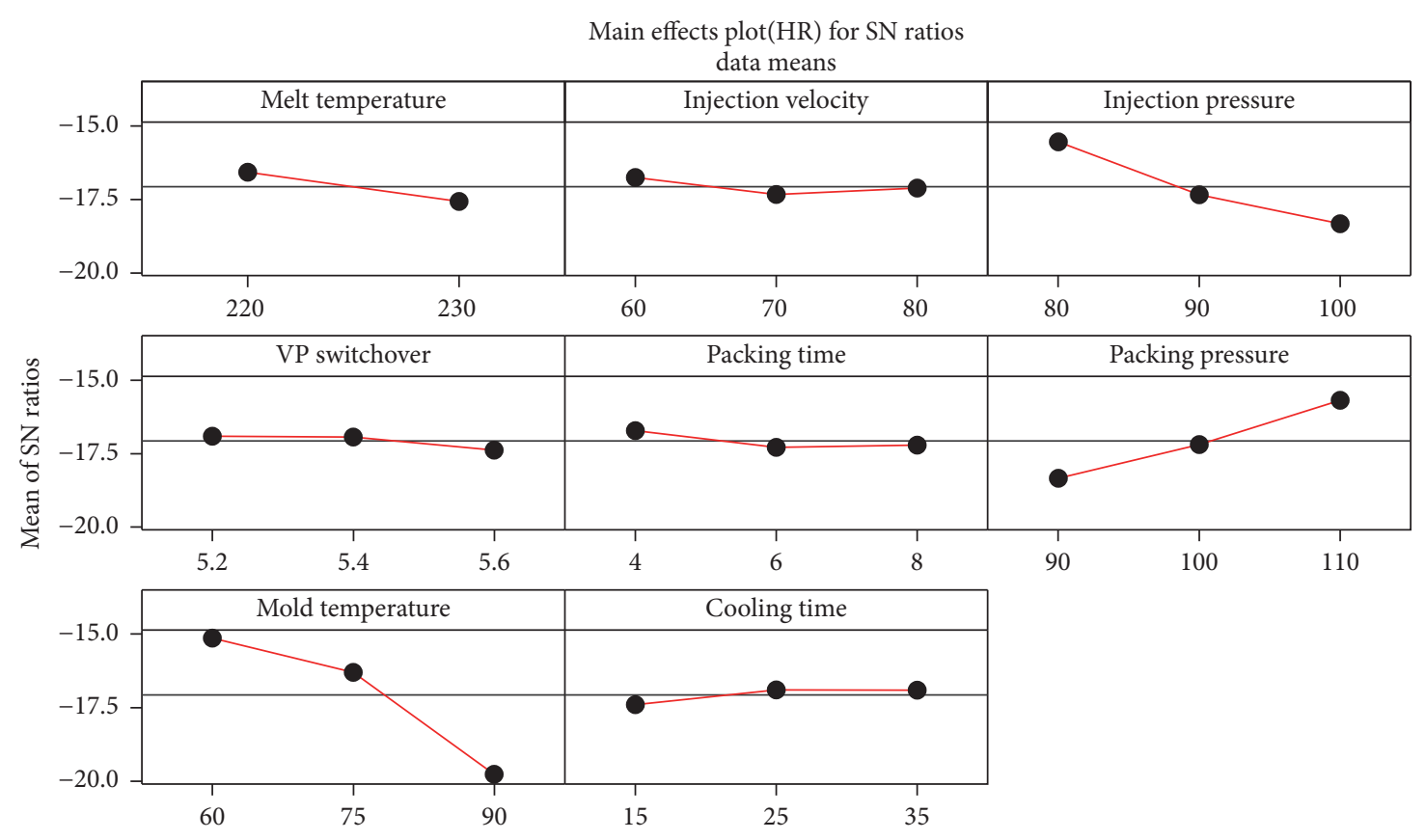

Signal-to-noise: smaller is better

FIGURE 4: Main effects plot (HR) for S/N ratios.

the global optimal value. In order to further improve the quality of optical lens, this paper established the mapping relationships between the key IMPP and objectives and conducted a global optimization using the multiobjective intelligent optimization algorithm, NSGA-II. The methods used for establishing the mapping relationships were M-SVR and BPNN algorithms, and their prediction abilities were compared. In this paper, the $3^{4}$ full factors experiment was conducted with injection pressure, packing pressure, molding temperature, and cooling time as key IMPP. The experimental results were used as training and test samples for M-SVR and BPNN. The IMPP that have no significant effect on the quality objectives were initialized to the mean value of best levels according to their $\mathrm{S} / \mathrm{N}$ ratios. For example, the best values of injection velocity for $\mathrm{HR}$ and $\mathrm{PV}_{20}$ were 60 and 70 , respectively, so its value was set to the 65 . In addition, it was necessary to adjust the level value for one of the key IMPP. From Figure 4, it can be seen that the injection pressure only has a great effect on the $\mathrm{HR}$ and that the $\mathrm{S} / \mathrm{N}$ ratios have a negative correlation; namely, the quality of optical lens decreases more quickly as a result of the increase in HR, with the increase in the injection pressure. Therefore, the level of injection pressure should be adjusted to smaller values. The IMPP and levels for $3^{4}$ full factorial experiments are shown in Table 5. Similarly, the experiments were arranged in random order, and the experimental results are shown in Table 6.

The M-SVR and BPNN models were built with the data of 81 samples as in Table 6 , of which 60 samples were randomly selected as training data, and those of the remaining 21 samples were used as test data. This paper used MATLAB R2014b as a modelling tool, the M-SVR algorithm was Libsvm toolbox developed by Chang and Lin [25], the kernel function was the radial basis function (RBF), and the performance goal was $1 E-4$. The Levenberg-Marquardt algorithm [26, 27] with a higher network convergence rate was selected in the BPNN algorithm, and the training variables were set as epochs 1000, 


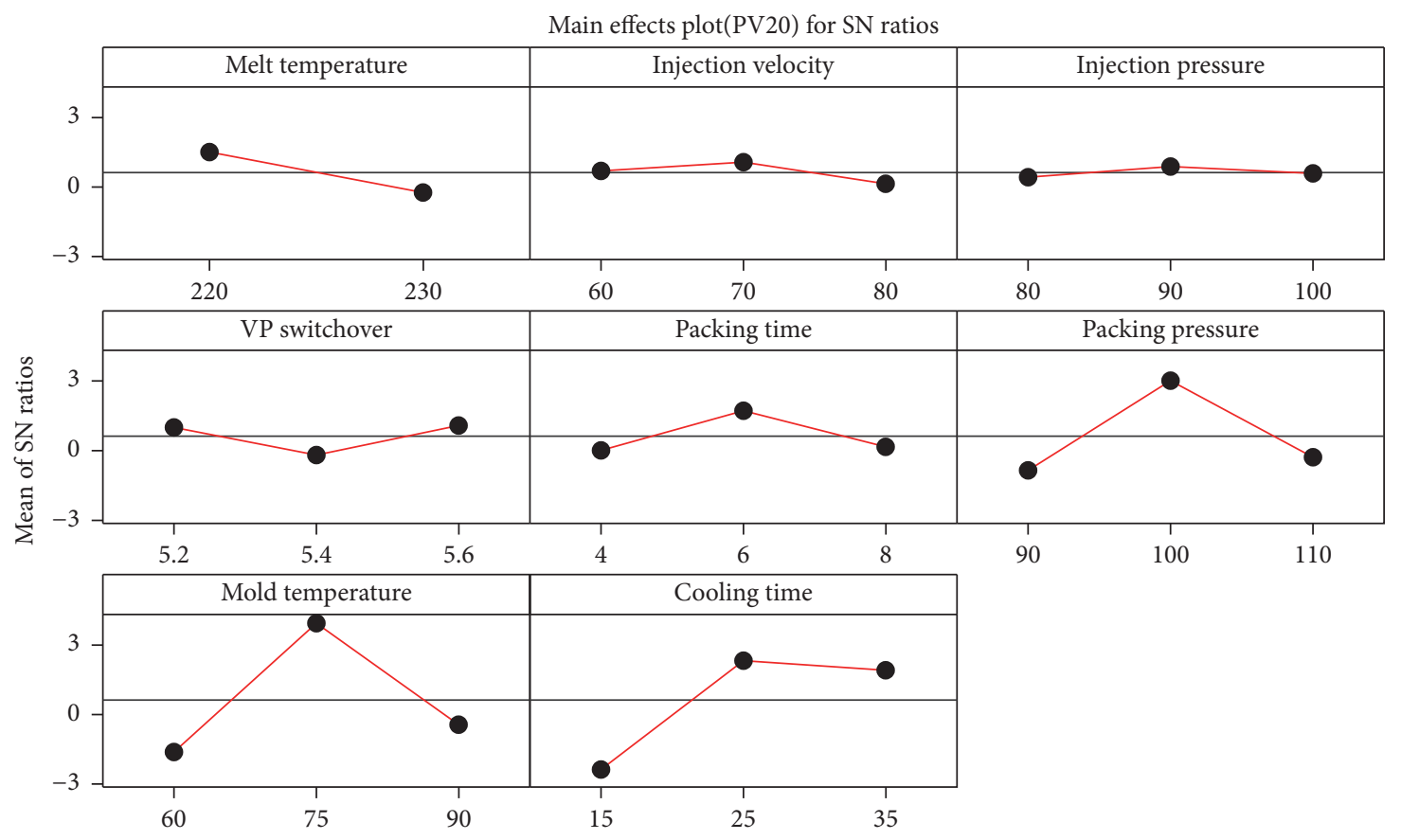

Signal-to-noise: smaller is better

FIgURE 5: Main effects plot $\left(\mathrm{PV}_{20}\right)$ for $\mathrm{S} / \mathrm{N}$ ratios.

TABLE 5: The IMPP and their levels for $3^{4}$ full factors experiment.

\begin{tabular}{lccc}
\hline IMPP & Level 1 & Level 2 & Level 3 \\
\hline A: melt temperature $\left({ }^{\circ} \mathrm{C}\right)$ & 220 & - & - \\
$B:$ injection velocity $(\mathrm{mm} / \mathrm{s})$ & 65 & - & - \\
$C:$ injection pressure $(\mathrm{MPa})$ & 60 & - & 80 \\
$D:$ VP switch over $(\mathrm{mm})$ & 5.5 & - & - \\
$E:$ packing time $(\mathrm{s})$ & 5 & 100 & 70 \\
$F:$ packing pressure $(\mathrm{MPa})$ & 90 & 75 & 90 \\
$G:$ mold temperature $\left({ }^{\circ} \mathrm{C}\right)$ & 60 & 25 & 35 \\
$H:$ cooling time $(\mathrm{s})$ & 15 & & - \\
\hline
\end{tabular}

performance goal $1 E-3$, learning rate 0.01 , and gradient $1 E-10$. The input variables of the BPNN and M-SVR were the significant IMPP discussed earlier, and the output variables were $\mathrm{HR}$ and $\mathrm{PV}_{20}$ of optical lens, where the BPNN model adopted a three-layer structure, and the number of neurons in the hidden layer was determined by experience or trialand-error method. Through experiments, when the number of neurons in the hidden layer was 7, the obtained BPNN model had the best prediction ability. In the training and testing stages of $\mathrm{HR}$ and $\mathrm{PV}_{20}$, the comparisons between the predicted values and actual values of BPNN algorithms and M-SVR algorithms were as shown in Figures 6, 7, 8, and 9. The determinant coefficients $R^{2}$ in the training and testing stages were as shown in Table 7. $R^{2}$ of the M-SVR model for $\mathrm{HR}$ and $\mathrm{PV}_{20}$ were 0.9841 and 0.9642 , respectively, in the training stages, and 0.9706 and 0.9591 , respectively, in the testing stage; these values were very close to 1 , indicating that the training data had good representation, and the training model accuracy was high. $R^{2}$ of the BPNN model were obviously smaller than those of the M-SVR model, indicating that the effect was not stable in the training of small samples. Especially in the testing stage, the prediction effort at some points was very large, completely unsuitable for precise prediction. Therefore, the M-SVR algorithm was selected to establish the relationships between the key IMPP and the two objectives.

4.3. Multiobjective Optimization. In this paper, the optical performance and surface waviness of the optical lens needed to be optimized at the same time; this belongs to the typical multiobjective optimization. The two objectives have the smaller-the-better characteristic. Therefore, the issue of multiobjective optimization is defined as follows:

Find: $\quad \mathbf{x}$

Minimize: $\quad F(\mathbf{x})=\left(f_{1}(\mathbf{x}), f_{2}(\mathbf{x})\right)$

Subject to: $\quad \mathbf{b}_{\text {lower }} \leq \mathbf{x} \leq \mathbf{b}_{\text {upper }}$, 
TABLE 6: $3^{4}$ full factorial experimental results.

\begin{tabular}{|c|c|c|c|c|c|c|}
\hline \multirow{2}{*}{ Number } & \multicolumn{4}{|c|}{ Key IPMM } & \multirow{2}{*}{ HR (\%) } & \multirow{2}{*}{$\mathrm{PV}_{20}(\mu \mathrm{m})$} \\
\hline & C & $F$ & $G$ & $H$ & & \\
\hline (1) & 60 & 110 & 75 & 15 & 6.81 & 0.923 \\
\hline (2) & 60 & 100 & 60 & 35 & 5.92 & 0.871 \\
\hline (3) & 80 & 110 & 75 & 25 & 5.11 & 0.721 \\
\hline (4) & 70 & 90 & 90 & 15 & 11.42 & 1.525 \\
\hline (5) & 70 & 90 & 60 & 25 & 7.91 & 1.279 \\
\hline (6) & 60 & 110 & 90 & 35 & 8.41 & 0.853 \\
\hline (7) & 80 & 110 & 90 & 15 & 8.35 & 1.674 \\
\hline (8) & 70 & 110 & 60 & 15 & 5.13 & 1.715 \\
\hline (9) & 70 & 90 & 75 & 35 & 8.21 & 0.651 \\
\hline (10) & 80 & 90 & 60 & 35 & 7.56 & 1.347 \\
\hline (11) & 80 & 90 & 60 & 25 & 5.93 & 1.217 \\
\hline (12) & 80 & 110 & 60 & 25 & 4.71 & 0.973 \\
\hline (13) & 80 & 100 & 75 & 15 & 6.32 & 0.818 \\
\hline (14) & 80 & 90 & 90 & 25 & 9.51 & 1.301 \\
\hline (15) & 60 & 110 & 75 & 25 & 4.91 & 0.723 \\
\hline (16) & 70 & 100 & 75 & 25 & 5.83 & 0.337 \\
\hline (17) & 70 & 100 & 60 & 25 & 5.83 & 0.826 \\
\hline (18) & 70 & 110 & 90 & 35 & 8.55 & 0.832 \\
\hline (19) & 80 & 110 & 75 & 15 & 7.05 & 0.921 \\
\hline (20) & 70 & 100 & 90 & 25 & 9.42 & 0.401 \\
\hline$(21)$ & 70 & 110 & 75 & 25 & 4.45 & 0.721 \\
\hline$(22)$ & 60 & 110 & 90 & 25 & 7.92 & 1.131 \\
\hline (23) & 70 & 100 & 60 & 15 & 5.98 & 1.273 \\
\hline (24) & 80 & 90 & 75 & 35 & 8.33 & 0.643 \\
\hline (25) & 70 & 110 & 90 & 25 & 8.05 & 1.142 \\
\hline (26) & 70 & 100 & 60 & 35 & 6.11 & 0.852 \\
\hline (27) & 80 & 100 & 60 & 25 & 5.92 & 0.833 \\
\hline (28) & 80 & 110 & 60 & 15 & 5.22 & 1.723 \\
\hline (29) & 60 & 90 & 60 & 15 & 7.47 & 1.472 \\
\hline (30) & 70 & 110 & 75 & 15 & 6.93 & 0.935 \\
\hline (31) & 70 & 90 & 60 & 15 & 7.83 & 1.521 \\
\hline$(32)$ & 70 & 100 & 75 & 15 & 6.2 & 0.802 \\
\hline (33) & 80 & 100 & 60 & 15 & 6.01 & 1.593 \\
\hline (34) & 60 & 110 & 60 & 15 & 5.02 & 1.732 \\
\hline (35) & 70 & 110 & 75 & 35 & 3.94 & 0.791 \\
\hline (36) & 70 & 110 & 60 & 35 & 4.92 & 1.213 \\
\hline (37) & 80 & 100 & 75 & 35 & 6.12 & 0.378 \\
\hline (38) & 70 & 90 & 75 & 25 & 8.25 & 0.507 \\
\hline (39) & 70 & 100 & 75 & 35 & 5.97 & 0.421 \\
\hline$(40)$ & 80 & 90 & 90 & 15 & 11.5 & 1.516 \\
\hline$(41)$ & 80 & 110 & 60 & 35 & 5.1 & 1.123 \\
\hline (42) & 80 & 90 & 60 & 15 & 4.83 & 1.824 \\
\hline (43) & 70 & 90 & 75 & 15 & 8.33 & 1.031 \\
\hline (44) & 70 & 90 & 60 & 35 & 7.45 & 1.34 \\
\hline (45) & 60 & 110 & 75 & 35 & 3.82 & 0.795 \\
\hline (46) & 80 & 90 & 90 & 35 & 10.21 & 1.358 \\
\hline (47) & 60 & 90 & 75 & 15 & 8.15 & 0.771 \\
\hline (48) & 70 & 110 & 60 & 25 & 4.59 & 0.978 \\
\hline
\end{tabular}

TABLE 6: Continued.

\begin{tabular}{|c|c|c|c|c|c|c|}
\hline \multirow{2}{*}{ Number } & \multicolumn{4}{|c|}{ Key IPMM } & \multirow{2}{*}{ HR (\%) } & \multirow{2}{*}{$\mathrm{PV}_{20}(\mu \mathrm{m})$} \\
\hline & $C$ & $F$ & $G$ & $H$ & & \\
\hline (49) & 60 & 100 & 75 & 15 & 6.08 & 0.821 \\
\hline (50) & 70 & 90 & 90 & 25 & 9.43 & 1.316 \\
\hline (51) & 60 & 90 & 60 & 35 & 7.32 & 1.343 \\
\hline (52) & 60 & 110 & 60 & 25 & 4.48 & 0.982 \\
\hline (53) & 60 & 110 & 90 & 15 & 8.21 & 1.379 \\
\hline (54) & 80 & 100 & 90 & 15 & 12.48 & 1.432 \\
\hline (55) & 60 & 90 & 90 & 25 & 9.32 & 1.325 \\
\hline (56) & 80 & 90 & 75 & 15 & 8.41 & 0.478 \\
\hline (57) & 80 & 100 & 60 & 35 & 6.23 & 0.842 \\
\hline (58) & 80 & 100 & 75 & 25 & 5.92 & 0.346 \\
\hline (59) & 60 & 100 & 90 & 15 & 11.89 & 1.431 \\
\hline$(60)$ & 60 & 90 & 75 & 35 & 8.1 & 0.655 \\
\hline (61) & 60 & 100 & 90 & 35 & 8.2 & 0.479 \\
\hline (62) & 70 & 100 & 90 & 15 & 11.97 & 1.415 \\
\hline (63) & 60 & 110 & 60 & 35 & 4.93 & 1.231 \\
\hline (64) & 60 & 90 & 75 & 25 & 8.13 & 0.501 \\
\hline (65) & 80 & 100 & 90 & 35 & 8.44 & 0.451 \\
\hline (66) & 60 & 100 & 90 & 25 & 9.92 & 0.581 \\
\hline (67) & 80 & 110 & 75 & 35 & 4.45 & 0.787 \\
\hline (68) & 60 & 90 & 90 & 15 & 11.35 & 1.502 \\
\hline (69) & 80 & 110 & 90 & 25 & 8.17 & 1.14 \\
\hline (70) & 70 & 110 & 90 & 15 & 8.31 & 1.739 \\
\hline$(71)$ & 60 & 100 & 60 & 25 & 5.72 & 0.852 \\
\hline (72) & 60 & 90 & 60 & 25 & 7.35 & 1.339 \\
\hline (73) & 80 & 110 & 90 & 35 & 8.64 & 0.836 \\
\hline (74) & 60 & 100 & 60 & 15 & 5.83 & 0.923 \\
\hline (75) & 70 & 100 & 90 & 35 & 8.33 & 0.462 \\
\hline (76) & 70 & 90 & 90 & 35 & 9.87 & 1.327 \\
\hline (77) & 80 & 90 & 75 & 25 & 8.36 & 0.503 \\
\hline (78) & 60 & 100 & 75 & 35 & 5.92 & 0.495 \\
\hline (79) & 60 & 100 & 75 & 25 & 5.71 & 0.334 \\
\hline (80) & 60 & 90 & 90 & 35 & 10.46 & 1.337 \\
\hline$(81)$ & 80 & 100 & 90 & 25 & 9.62 & 0.467 \\
\hline
\end{tabular}

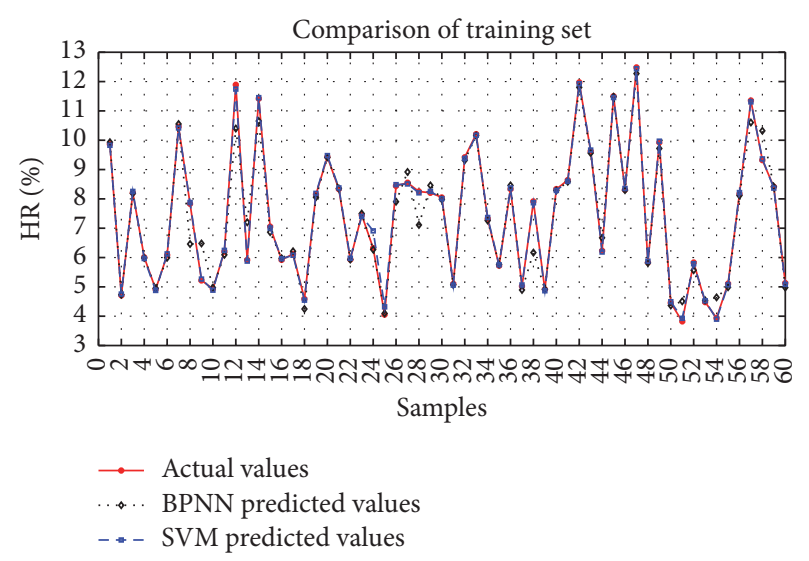

FIGURE 6: Comparison of HR prediction results in training set. 
TABLE 7: The $R^{2}$ of BPNN model and M-SVR model at training and testing stage.

\begin{tabular}{lcccc}
\hline \multirow{2}{*}{ Objective } & \multicolumn{2}{c}{ Training } & \multicolumn{2}{c}{ Testing } \\
& BPNN & M-SVR & BPNN & M-SVR \\
\hline HR & 0.8984 & 0.9841 & 0.7824 & 0.9706 \\
PTV & 0.8822 & 0.9642 & 0.8255 & 0.9591 \\
\hline
\end{tabular}
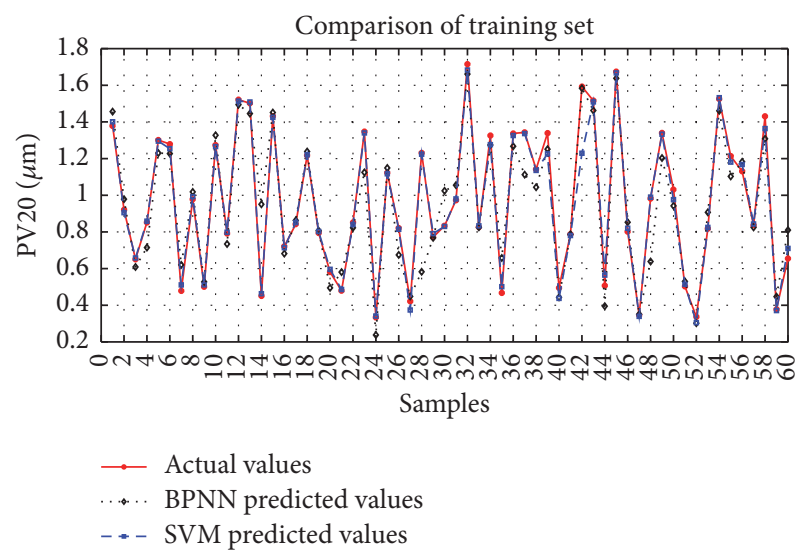

FIGURE 7: Comparison of $\mathrm{PV}_{20}$ prediction results in training set.

where $f_{1}(\mathbf{x})$ and $f_{2}(\mathbf{x})$ denote $\mathrm{HR}$ and $\mathrm{PV}_{20}$, respectively. $\mathbf{x}$ is the key IMPP vector $(C F G H)^{T}$. According to Table 5, it is known that $\mathbf{b}_{\text {lower }}=\left(\begin{array}{llll}60 & 90 & 60 & 15\end{array}\right)^{T}$ and $\mathbf{b}_{\text {upper }}=$ $\left(\begin{array}{llll}80 & 110 & 90 & 35\end{array}\right)^{T}$. There are many effective methods to solve multiobjective optimization problems, in which the traditional linear weighted method (LWM) and the popular NSGA-II algorithms are representative. In order to compare the optimization effects of these two methods, they have been tested separately in MATLAB R2014b. LWM is the method that can transform multiobjective optimization into single objective optimization. Each objective function was weighted and then summed up. Since the two objective functions have different units, they need to be dimensionless. The value of weights can reflect the importance of $f(\mathbf{x})$, and each set of weights, $\omega_{1}$ and $\omega_{2}$, can obtain an optimized key IMPP by minimizing $F(\mathbf{x})$. In this paper, one objective reflects the optical performance, while the other reflects the surface waviness. Theoretically speaking, if the two objectives are equally important, the weights should be set as $\omega_{1}=\omega_{2}=0.5$. In order to explore the overall optimization effect of LWM, 9 sets of weights have been set for testing. Using constrained nonlinear minimization Solver as the optimization tool, the operating parameters were set as follows: $\max$ iterations = 1500 , function tolerance $=1 E-5$, and the other stopping criteria were set as default value. The optimal key IMPP and two objectives have been achieved, as shown in Table 8 . Figure 10 clearly shows that there is a dominating relationship between some optimization points. For example, under the condition of $\boldsymbol{\omega}=\left(\omega_{1} \omega_{2}\right)=\left(\begin{array}{ll}0.9 & 0.1\end{array}\right)$, the optimal solution is inferior to the optimal solution under the conditions of $\boldsymbol{\omega}=(0.70 .3),(0.60 .4)$. Therefore, if the weight of HR is greater, the optimization result can be unreliable. This is

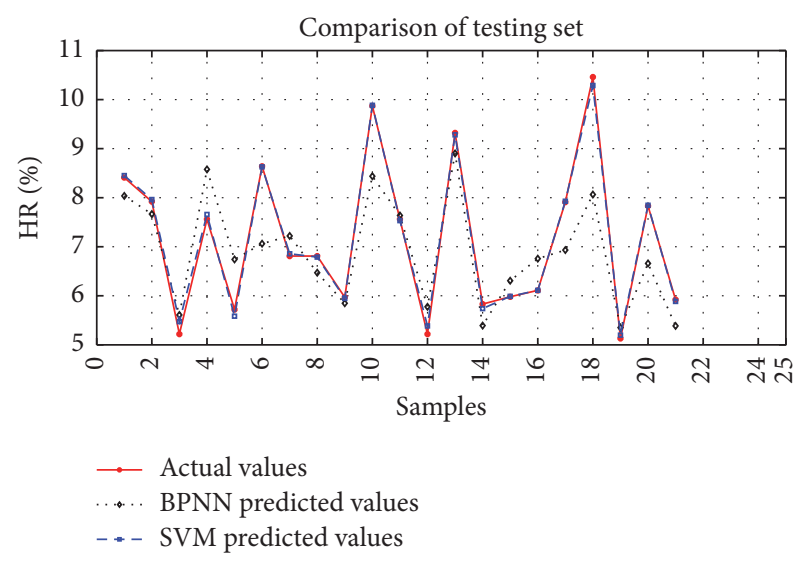

FIGURE 8: Comparison of HR prediction results in testing set.

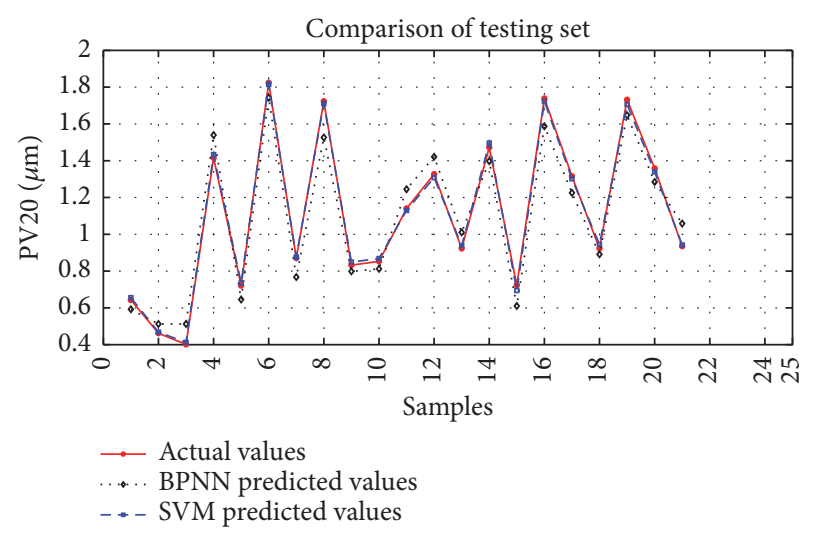

FIgURE 9: Comparison of $\mathrm{PV}_{20}$ prediction results in testing set.

mainly because the key IMPP and the two objectives have strong nonlinear relations, and $F(\mathbf{x})$ is a nonconvex function. In fact, the optimal solution is highly likely to be the local optimum.

The NSGA-II algorithm has excellent multiobjective optimization ability. The functions $f_{1}(\mathbf{x}), f_{2}(\mathbf{x})$ established using the M-SVR algorithm were taken as the two fitness functions of the NSGA-II algorithm. The running parameters were set as follows: population size 100, maximum number of generations 200, stall generation limits 200, tolerance of fitness function $1 E-100$, mutation probability 0.25 , crossover probability 0.8 , and Pareto fraction 0.1. The running results of the NSGA-II algorithm are shown in Figure 10. The Pareto-optimal set has $100 \times 0.1=10$ individuals. After the approximate treatment was performed on the optimized IMPP and the corresponding objectives, the results are as shown in Table 9. The range of HR is 3.826 to 5.766 and that of $\mathrm{PV}_{20}$ is 0.347 to 0.791 . As can be seen from Table 6, the known minimum values of $\mathrm{HR}$ and $\mathrm{PV}_{20}$ are 3.82 and 0.334 , respectively. The Pareto-optimal set does not contain the case in which the two objectives are minimum, indicating there is a conflict between the two objectives and the optimal status cannot be achieved at the same time. Figure 10 further shows that $\mathrm{PV}_{20}$ gradually increases with the decrease of $\mathrm{HR}$. In contrast to LWM and NSGA-II, all the Pareto-optimal 
TABLE 8: The Pareto-optimal set for $H R$ and $\mathrm{PV}_{20}$ using LWM.

\begin{tabular}{|c|c|c|c|c|c|c|c|}
\hline \multicolumn{2}{|c|}{ Weight } & \multicolumn{4}{|c|}{ Key IMPP } & \multirow{2}{*}{ HR (\%) } & \multirow{2}{*}{$\mathrm{PV}_{20}(\mu \mathrm{m})$} \\
\hline$\omega_{1}$ & $\omega_{2}$ & $C$ & $F$ & $G$ & $H$ & & \\
\hline 0.9 & 0.1 & 61.3 & 108.8 & 75.6 & 36.6 & 4.226 & 0.852 \\
\hline 0.8 & 0.2 & 63.6 & 108.5 & 74.8 & 33.5 & 4.552 & 0.661 \\
\hline 0.7 & 0.3 & 60.2 & 109.1 & 76.1 & 26.4 & 4.221 & 0.602 \\
\hline 0.6 & 0.4 & 66.4 & 91.3 & 73.4 & 28.4 & 4.425 & 0.512 \\
\hline 0.5 & 0.5 & 63.1 & 107.5 & 77.9 & 27.8 & 4.612 & 0.491 \\
\hline 0.4 & 0.6 & 64.5 & 106.6 & 72.5 & 28.3 & 4.774 & 0.488 \\
\hline 0.3 & 0.7 & 65.3 & 99.7 & 72.4 & 26.1 & 5.312 & 0.456 \\
\hline 0.2 & 0.8 & 66.6 & 101.2 & 76.0 & 25.6 & 5.133 & 0.421 \\
\hline 0.1 & 0.9 & 66.2 & 105.8 & 78.3 & 25.4 & 5.559 & 0.404 \\
\hline
\end{tabular}

TABle 9: The Pareto-optimal set for $\mathrm{HR}$ and $\mathrm{PV}_{20}$ using NSGA-II.

\begin{tabular}{|c|c|c|c|c|c|c|}
\hline \multirow{2}{*}{ Number } & \multicolumn{4}{|c|}{ Key IMPP } & \multirow{2}{*}{ HR (\%) } & \multirow{2}{*}{$\mathrm{PV}_{20}(\mu \mathrm{m})$} \\
\hline & $C$ & $F$ & G & $H$ & & \\
\hline (1) & 60.6 & 109.6 & 73.8 & 34.6 & 3.826 & 0.791 \\
\hline$(2)$ & 61.3 & 109.1 & 74.6 & 32.6 & 4.135 & 0.601 \\
\hline (3) & 61.6 & 107.9 & 75.5 & 28.6 & 4.216 & 0.553 \\
\hline$(4)$ & 62.3 & 95.7 & 75.8 & 27.7 & 4.385 & 0.502 \\
\hline (5) & 62.9 & 105.3 & 75.9 & 26.8 & 4.451 & 0.486 \\
\hline (6) & 63.5 & 104.6 & 76.6 & 26.1 & 4.512 & 0.473 \\
\hline (7) & 64.1 & 98.6 & 76.4 & 25.8 & 4.762 & 0.432 \\
\hline (8) & 64.6 & 102.3 & 76.8 & 24.4 & 4.944 & 0.406 \\
\hline (9) & 65.2 & 101.4 & 76.7 & 25.4 & 5.267 & 0.384 \\
\hline$(10)$ & 65.7 & 100.3 & 76.8 & 25.2 & 5.766 & 0.347 \\
\hline
\end{tabular}

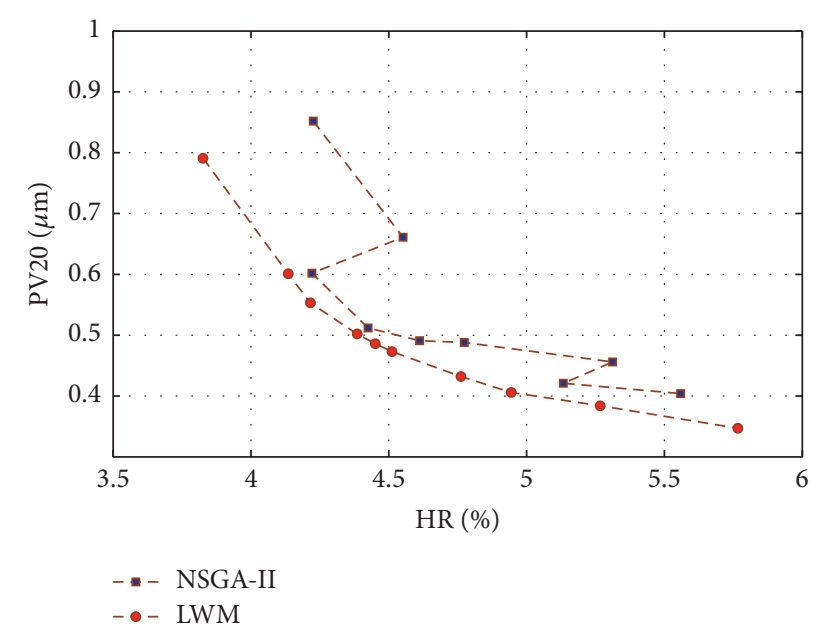

FIgure 10: Pareto-optimal set for $\mathrm{HR}$ and $\mathrm{PV}_{20}$.

set of the key IMPP obtained by NSGA-II can dominate the one obtained by LWM. Therefore, NSGA-II algorithm has apparent advantages in dealing with conflicting and nonconvex multiobjective optimization problems.

4.4. Confirm Experiment. In order to verify the validity of the optimization results obtained by NSGA-II, the oddnumber optimization results in Table 9 were selected for the experiment. The objective values predicted by the NSGA-II algorithm and the experimental results were compared to quantitatively determine the reliability of optimization. This paper took the mean prediction error (MPE) as an evaluating indicator, and the formula used is as follows:

$$
\mathrm{MPE}=\frac{1}{n} \sum_{i=1}^{n} \frac{\left|y_{i}-\widetilde{y}_{i}\right|}{y_{i}} \times 100 \%,
$$

where $y_{i}$ is the result of the $i$ th experiment on the objectives, $\widetilde{y_{i}}$ is the result of the $i$ th prediction of the objectives, and $n$ is the total number of experiments. The results of the verification are shown in Table 10 . The MPE of $H R$ and $P_{20}$ are $7.16 \%$ and $9.78 \%$, respectively, and the average error is less than $10 \%$, which indicates that the optimization procedures presented by this paper have good prediction accuracy. In this paper, the material of optical lens is PMMA-HT50Y, and the tolerance of products for $\mathrm{HR}$ and $\mathrm{PV}_{20}$ is $0.5 \%$ and $0.05 \mu \mathrm{m}$, respectively. According to Tables 9 and 10, the average value of $\mathrm{HR}$ and $\mathrm{PV}_{20}$ is $4.626 \%$ and $0.498 \mu \mathrm{m}$, respectively. The fluctuation of error is $0.0716 \times 4.626 \%=0.331 \%$ for $\mathrm{HR}$, and $0.0978 \times 0.498 \mu \mathrm{m}=0.049 \mu \mathrm{m}$ for $\mathrm{PV}_{20}$. Therefore, the fluctuation of error is within the tolerance range of the products, and the NSGA-II algorithm can provide guidance for actual production. Finally, the optimization results can be selected from a Pareto-optimal set according to the bias of the two quality objectives. For example, the process parameters 
TABLE 10: Confirmation experimental results for Pareto-optimal set of $H R$ and $\mathrm{PV}_{20}$.

\begin{tabular}{|c|c|c|c|c|c|c|c|c|}
\hline \multirow{2}{*}{ Number } & \multicolumn{4}{|c|}{ Key IMPP } & \multicolumn{2}{|c|}{ Experimental results } & \multicolumn{2}{|c|}{ Prediction error } \\
\hline & C & $F$ & G & $H$ & $\operatorname{HR}(\%)$ & $\mathrm{PV}_{20}(\mu \mathrm{m})$ & $\operatorname{HR}(\%)$ & $\mathrm{PV}_{20}(\%)$ \\
\hline (1) & 60.6 & 109.6 & 73.8 & 34.6 & 3.531 & 0.712 & 8.35 & 11.10 \\
\hline (3) & 61.6 & 107.9 & 75.5 & 28.6 & 3.912 & 0.506 & 7.77 & 9.29 \\
\hline (5) & 62.9 & 105.3 & 75.9 & 26.8 & 4.226 & 0.532 & 5.32 & 8.65 \\
\hline (7) & 64.1 & 98.6 & 76.4 & 25.8 & 5.141 & 0.479 & 7.37 & 9.81 \\
\hline (9) & 65.2 & 101.4 & 76.7 & 25.4 & 4.924 & 0.349 & 6.97 & 10.03 \\
\hline MPE & & & & & & & 7.16 & 9.78 \\
\hline
\end{tabular}

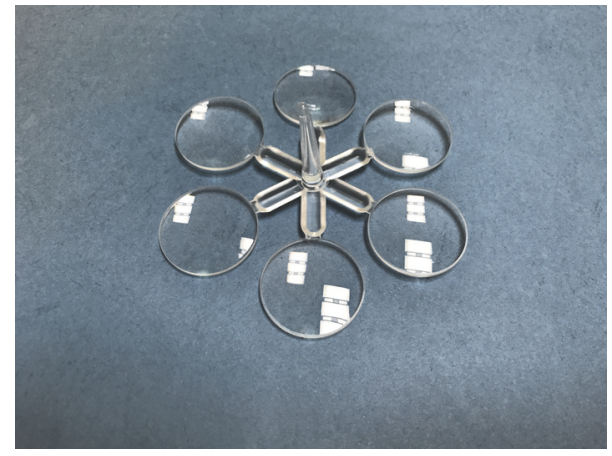

FIGURE 11: The injection plastic optical lens.

combination of Experiment number 5 was selected, and the injection plastic optical lens is shown in Figure 11.

\section{Conclusion}

In order to consider the optical performance and surface waviness of the plastic optical lens at the same time, this paper presents a set of optimization procedures with four stages, taking $\mathrm{HR}$ and $\mathrm{PV}_{20}$ as the quality objectives. The NSGA-II algorithm was applied to solve this multioptimization problem. In particular, the introduction of the M-SVR algorithm as the fitness function played an important role, through which we improved the prediction accuracy of the quality objectives. The Pareto-optimal set obtained in this study improved the quality of plastic optical lens comprehensively. The conclusions are as follows:

(1) The IMPP having a significant effect on both objectives, $\mathrm{HR}$ and $\mathrm{PV}_{20}$, include packing pressure and molding temperature. The IMPP only having a significant effect on HR is injection pressure. With the increase in injection pressure, the optical performance of the lens decreases continuously. The IMPP only having a significant effect on $\mathrm{PV}_{20}$ is cooling time. With the increase in cooling time, the lens surface waviness was increased substantially at the beginning and decreased slightly subsequently.

(2) When establishing the relationships between the objectives and the key IMPP, the $3^{4}$ full factorial experimental results of the IMPP were used as samples. At the same time, the BPNN algorithm and the M-SVR algorithm were used to establish the relationships. The results show that the determinant coefficients $R^{2}$ of the M-SVR model, which are close to 1 , are bigger than that of the BPNN model in the training and testing stages, indicating that the M-SVR algorithm has high prediction accuracy when dealing with low-dimensional small samples.

(3) Using the NSGA-II algorithm can effectively find the Pareto-optimal set of IMPP, so that the two objectives $\mathrm{HR}$ and $\mathrm{PV}_{20}$ are as small as possible, to achieve the optimal balance. Through the verification on the optimization results, the MPE of $\mathrm{HR}$ and $\mathrm{PV}_{20}$ reached a high accuracy of $7.16 \%$ and $9.78 \%$, respectively. Finally, the optimization results may be selected from the Pareto-optimal set according to the bias of the two quality objectives.

\section{Conflicts of Interest}

The authors declare that they have no conflicts of interest.

\section{Acknowledgments}

This research is supported by Science and Technology Planning Project of Guangdong Province under Grants nos. 2015B090921007 and 2015B010919001.

\section{References}

[1] M. Pazos, J. Baselga, and J. Bravo, "Limiting thickness estimation in polycarbonate lenses injection using CAE tools," Journal of Materials Processing Technology, vol. 143-144, no. 1, pp. 438441, 2003.

[2] J. Wen and P. Wen, "Simulation and optimization of aspheric plastic lens injection molding," Journal of Wuhan University of Technology-Mater. Sci. Ed., vol. 20, no. 2, pp. 86-89, 2005.

[3] R. J. Bensingh, S. R. Boopathy, and C. Jebaraj, "Minimization of variation in volumetric shrinkage and deflection on injection molding of Bi-aspheric lens using numerical simulation," Journal of Mechanical Science and Technology, vol. 30, no. 11, pp. 5143-5152, 2016.

[4] J.-Y. Shieh, L. K. Wang, and S.-Y. Ke, "A feasible injection molding technique for the manufacturing of large diameter aspheric plastic lenses," Optical Review, vol. 17, no. 4, pp. 399403, 2010. 
[5] G.-H. Hu and Z.-S. Cui, "Effect of packing parameters and gate size on shrinkage of aspheric lens parts," Journal of Shanghai Jiaotong University (Science), vol. 15, no. 1, pp. 84-87, 2010.

[6] X. Lu and L. S. Khim, "A statistical experimental study of the injection molding of optical lenses," Journal of Materials Processing Technology, vol. 113, no. 1-3, pp. 189-195, 2001.

[7] R. Spina, P. Walach, J. Schild, and C. Hopmann, "Analysis of lens manufacturing with injection molding," International Journal of Precision Engineering and Manufacturing, vol. 13, no. 11, pp. 2087-2095, 2012.

[8] K.-M. Tsai, C.-Y. Hsieh, and W.-C. Lo, "A study of the effects of process parameters for injection molding on surface quality of optical lenses," Journal of Materials Processing Technology, vol. 209, no. 7, pp. 3469-3477, 2009.

[9] K.-M. Tsai and H.-J. Luo, "An inverse model for injection molding of optical lens using artificial neural network coupled with genetic algorithm," Journal of Intelligent Manufacturing, vol. 28, no. 2, pp. 473-487, 2017.

[10] K.-M. Tsai and J.-K. Lan, "Correlation between runner pressure and cavity pressure within injection mold," The International Journal of Advanced Manufacturing Technology, vol. 79, no. 1-4, pp. 273-284, 2015.

[11] S. Y. Kim, M. H. Rim, W. S. Lim, and W. Y. Kim, "A numerical approach for predicting the optical characteristics of injectionmolded lenses," Journal of Injection Molding Technology, vol. 4, no. 1, pp. 29-35, 2000.

[12] P. J. Wang and H. E. Lai, "Study of residual birefringence in injection molded lenses," in Proceedings of the ANTEC, pp. 2494-2498, 2007.

[13] L.-S. Turng, M. Peić, and D. K. Bradley, "Process simulation and optimization for injection molding - Experimental verifications and field applications," Journal of Injection Molding Technology, vol. 6, no. 2, pp. 143-155, 2002.

[14] J.-L. Kuo and M.-T. Chang, "Multiobjective Design of Turbo Injection Mode for Axial Flux Motor in Plastic Injection Molding Machine by Particle Swarm Optimization," Mathematical Problems in Engineering, vol. 2015, Article ID 974624, 2015.

[15] J. Zhao, G. Cheng, S. Ruan, and Z. Li, "Multi-objective optimization design of injection molding process parameters based on the improved efficient global optimization algorithm and nondominated sorting-based genetic algorithm," The International Journal of Advanced Manufacturing Technology, vol. 78, no. 9-12, pp. 1813-1826, 2015.

[16] X.-H. Yin, C. Yang, and X.-P. Li, "Simultaneous Control of Birefringence and Warpage for Thermoplastic Optical Lenses Fabricated Using Microinjection Molding," Polymer-Plastics Technology and Engineering, vol. 54, no. 17, pp. 1772-1779, 2015.

[17] M. Wang, J. Dong, W. Wang et al., "Optimal design of medium channels for water-assisted rapid thermal cycle mold using multi-objective evolutionary algorithm and multiattribute decision-making method," The International Journal of Advanced Manufacturing Technology, vol. 68, no. 9-12, pp. 24072417, 2013.

[18] X. Kong, Y. Yang, X. Chen, Z. Shao, and F. Gao, "Quality control via model-free optimization for a type of batch process with a short cycle time and low operational cost," Industrial \& Engineering Chemistry Research, vol. 50, no. 5, pp. 2994-3003, 2011.

[19] S. Zhu, Y. Yang, B. Yang, Z. Shao, and X. Chen, "Model-free quality optimization strategy for a batch process with short cycle time and low operational cost," Industrial \& Engineering Chemistry Research, vol. 53, no. 42, pp. 16384-16396, 2014.
[20] Y. Yang, B. Yang, S. Zhu, and X. Chen, "Online quality optimization of the injection molding process via digital image processing and model-free optimization," Journal of Materials Processing Technology, vol. 226, pp. 85-98, 2015.

[21] G. Taguchi, S. Chowdhury, and Y. Wu, Taguchis quality engineering handbook, Wiley, Hoboken, NJ, USA, 2005.

[22] D. E. Rumelhart, G. E. Hinton, and R. J. Williams, "Learning representations by back-propagating errors," Nature, vol. 323, no. 6088, pp. 533-536, 1986.

[23] F. Pérez-Cruz, G. Camps-Valls, E. Soria-Olivas, J. J. PérezRuixo, A. R. Figueiras-Vidal, and A. Artés-Rodríguez, "Multidimensional function approximation and regression estimation," in Artificial Neural Networks-ICANN 2002, vol. 2415 of Lecture Notes in Computer Science, pp. 757-762, Springer, Berlin, Germany, 2002.

[24] K. Deb, A. Pratap, S. Agarwal, and T. Meyarivan, "A fast and elitist multiobjective genetic algorithm: NSGA-II," IEEE Transactions on Evolutionary Computation, vol. 6, no. 2, pp. 182197, 2002.

[25] C.-C. Chang and C.-J. Lin, "Training v-support vector regression: Theory and algorithms," Neural Computation, vol. 14, no. 8, pp. 1959-1977, 2002.

[26] K. Levenberg, "A method for the solution of certain non-linear problems in least squares," Quarterly of Applied Mathematics, vol. 2, pp. 164-168, 1944.

[27] D. W. Marquardt, "An Algorithm for Least-Squares Estimation of Nonlinear Parameters," Journal of the Society for Industrial \& Applied Mathematics, vol. 11, no. 2, pp. 431-441, 2006. 


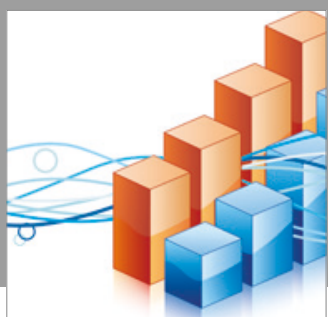

Advances in

Operations Research

vatersals

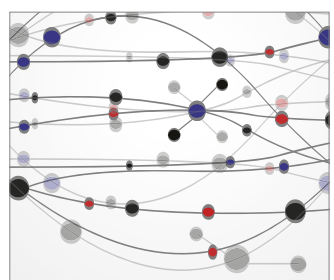

\section{The Scientific} World Journal
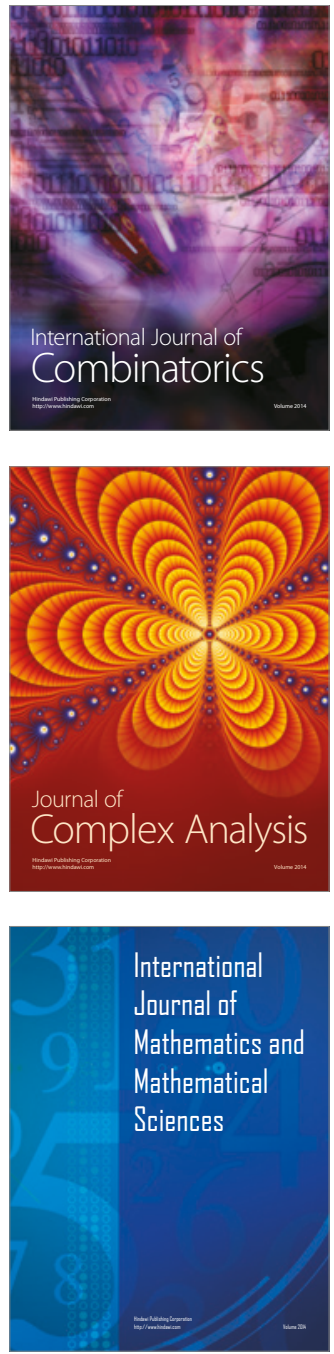
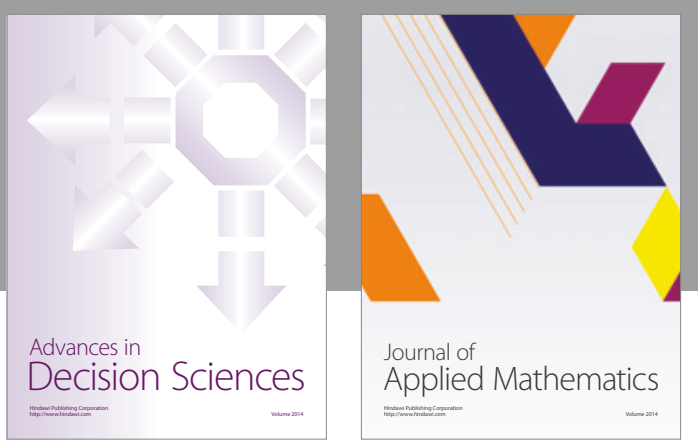

Algebra

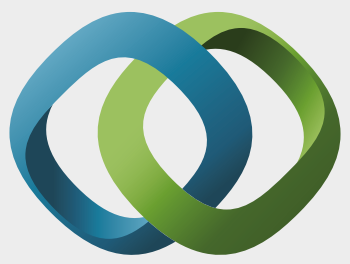

\section{Hindawi}

Submit your manuscripts at

https://www.hindawi.com
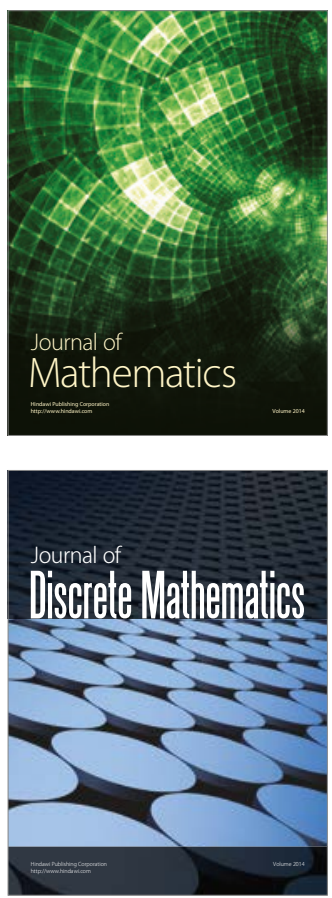

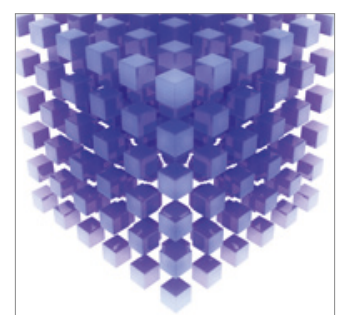

Mathematical Problems in Engineering
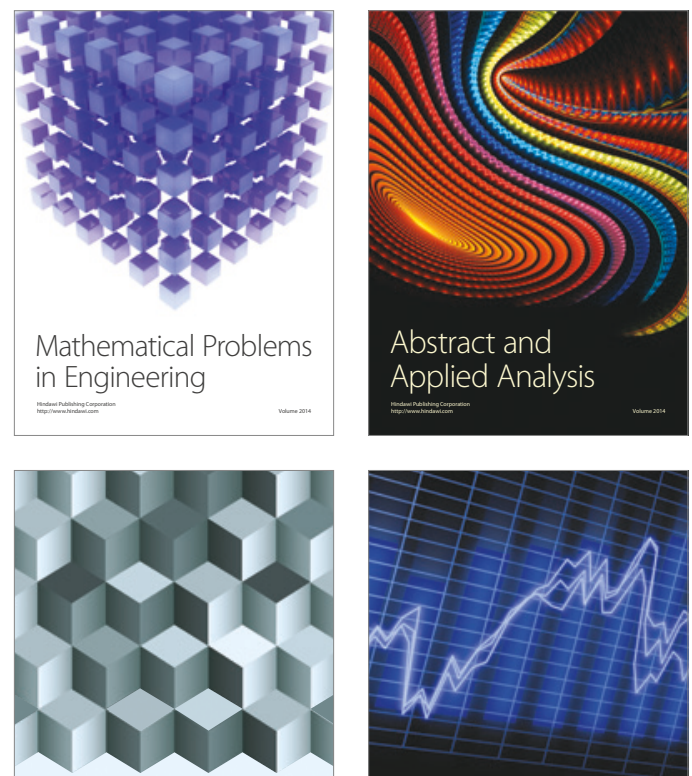

Journal of

Function Spaces

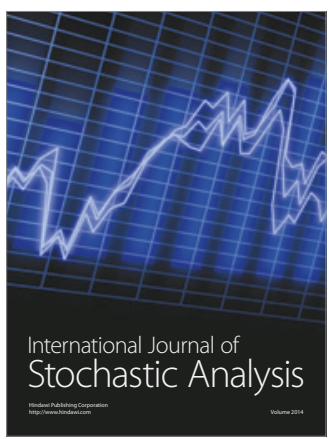

Probability and Statistics
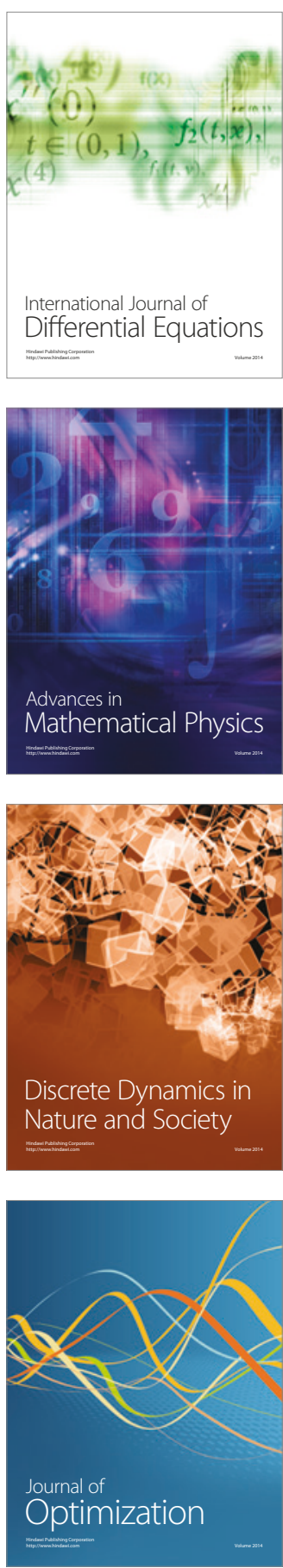